\title{
The influence of age on aerosol deposition in children with cystic fibrosis
}

\author{
H.L. Chua*, G.G. Collis*, A.M. Newbury**, K. Chan+, G.D. Bower**, \\ P.D. Slyt+, P.N. Le Souef*
}

The influence of age on aerosol deposition in children with cystic fibrosis. H.L. Chua, G.G. Collis, A.M. Newbury, K. Chan, G.D. Bower, P.D. Sly, P.N. Le Souef. CERS Journals 1994.

ABSTRACT: Nebulized aerosols are commonly used to deliver drugs for the treatment of respiratory disease in children, but there are inadequate data on the dose of drug depositing in the lungs in this age group, and the effect of age on this dose. We therefore aimed to quantify total and regional deposition of nebulized aerosol in children of widely differing age.

Twelve infants (median age $0.8 \mathrm{yrs}$, range $0.3-1.4 \mathrm{yrs}$ ) who were asleep, and eight older children (median age 10.8 yrs, range 6.3-18.0 yrs) with cystic fibrosis were studied. Radiolabelled normal saline aerosol was generated by a Turret nebulizer, with a driving flow of $9 l \cdot \mathrm{min}^{-1}$. All subjects inhaled aerosol via the nasal route, whilst the older children undertook a second study with inhalation via the oral route. Following aerosol inhalation, planar and single-photon emission computed tomography (SPECT) scans were obtained.

For the nasal route, total lung deposition was lower in infants (median 1.3\%, range $0.3-1.6 \%$ ) than in older children (median 2.7\%, range 1.6-4.4\%). For the older children inhaling via the nasal or oral route, there was no influence of age on lung, upper respiratory tract, or the sum of upper respiratory tract and lung deposition.

We conclude that the dose of a nasally inspired aerosol reaching the lungs of infants who are asleep is approximately half that for older children, when the nebulizer is operating at $9 \mathrm{l} \cdot \mathrm{min}^{-1}$. Age does not affect deposition of nasally or orally inspired aerosols in older children.

Eur Respir J., 1994, 7, 2185-2191
Depts of *Respiratory Medicine and **Nuclear Medicine, Princess Margaret Hospital for Children, Perth, Western Australia. 'Department of Pharmacy, University of Sydney, Sydney, and *Division of Clinical Sciences, Western Australian Research Institute for Child Health, Perth, Western Australia.

Correspondence: H. L. Chua Dept of Respiratory Medicine Princess Margaret Hospital for Children GPO Box D184

Perth 6001

Western Australia

Keywords: Aerosol deposition children

age

Received: March 241994

Accepted after revision July 311994
Nebulized aerosols are commonly used to deliver drugs to children's lungs. However, data on dose of drug depositing in the lung in children are sparse, and there is no consistent approach to calculation of the dose of aerosolized agents. Some drugs are delivered with the same concentration in the nebulizer solution for children of all ages, whereas others are given according to a weight-corrected concentration [1].

Inspiratory flow measurements by CoLLIS et al. [2] demonstrated that the inspiratory flow of children over approximately $1 \mathrm{yr}$ of age is likely to exceed nebulizer flow. This suggests that beyond infancy, all children will inhale the entire nebulizer output during inspiration and, therefore, inspire the same dose. The inspiratory flows of young infants are usually less than nebulizer flow [2], and all the available nebulizer output during inspiration cannot be inspired, resulting in a lower dose.

Mathematical models based on morphometric data have been used by many investigators, including $\mathrm{Yu}$ and Xu [3], Phalen and co-workers [4, 5], and Hofmann et al. $[6,7]$, to predict deposition patterns in children, but there have been no adequate in vivo studies addressing the influence of age on aerosol deposition in children. To allow more rational aerosol dosage regimens for clinical and research purposes in children, knowledge of total and regional deposition at different ages is essential. This information is especially important in children with cystic fibrosis, because nebulized antibiotics are now commonly relied upon for both acute and long-term therapy, and new therapeutic modalities, such as recombinant human deoxyribonuclease rhDNase [8] and gene therapy [9], are rapidly being developed.

We, therefore, aimed to quantify deposition in children with cystic fibrosis from infancy to late adolescence.

\section{Methods}

Twelve infants and eight older children with cystic fibrosis were recruited from Princess Margaret Hospital for Children. The seven male and five female infants had a median age of 0.8 yrs (range $0.3-1.4$ yrs) and mean forced expiratory volume in $0.5 \mathrm{~s}\left(\mathrm{FEV}_{0.5}\right)$ of $88 \%$ predicted (range $67-105 \% ; n=11$ ). The three male and five 
female older children had a median age of $10.8 \mathrm{yrs}$ (range 6.3-18.0 yrs) and mean forced expiratory volume in one second $\left(\mathrm{FEV}_{1}\right)$ of $101 \%$ predicted (range $70-120 \% ; n=8)$. Children aged $1.5-6$ yrs were not included, because it would have been difficult to adhere to the study protocol and induce sleep in this age group. We chose subjects who were clinically well at the time of the studies and had normal or near normal lung function. The study was approved by the Medical Ethics Committee of Princess Margaret Hospital for Children, and written, informed consent was obtained from the parents, and subjects where appropriate, before the studies were undertaken.

Radiolabelled normal saline $(0.9 \%$ with $15 \mathrm{mCi}$ of 99mtechnetium bound to diethylenetriamine penta-acetic acid (DTPA) (Amerscan Pentetate II, Amersham, UK)) was delivered by a Turret (Medic-Aid Ltd, UK) nebulizer (modified to prevent radioactive aerosol leakage into room air). The breathing circuit consisted of a Y-piece, an inspiratory limb with a one-way Hans Rudolph valve (Hans Rudolph Inc., Kansas City, Mo, USA), an expiratory limb with one-way Hans Rudolph valve and an absolute Barrier-Bac filter (DAR, Mirandola, Italy), and a facemask or mouthpiece. If the inspiratory flow of the subject exceeded nebulizer flow, air was entrained via the inspiratory limb at one arm of the Y-piece. Expired air entered the expiratory limb at another arm of the Ypiece and radioaerosol was trapped by the absolute filter. The facemask or mouthpiece was attached to the third arm of the Y-piece. The nebulizer was driven by compressed air at $9 \mathrm{l} \cdot \mathrm{min}^{-1}$ to produce a respirable aerosol [10], which entered the Y-piece where the three arms joined. The compressed airflow was fixed for all ages, because this would be the case in the usual clinical setting. Aerosol particle size of the radiolabelled normal saline used in the study (after decay of the isotope) was determined in the stream emerging from the circuit by a Malvern 2600 HSD laser particle analyser (Malvern Instruments, Malvern, UK); mass median aerodynamic diameter (MMAD) was $2.3 \mu \mathrm{m}$ and the geometric standard deviation (GSD) was 2.3.

\section{Protocol for infants}

The infants were induced to sleep with oral chloral hydrate $\left(80 \mathrm{mg} \cdot \mathrm{kg}^{-1}\right)$ on two occasions. Sleep was induced because a crying and struggling infant would not tolerate the study protocol and a good seal would not be obtained, resulting in some of the nebulizer dose not being inhaled and contamination of the atmosphere by radioactivity.

On one visit, $\mathrm{FEV}_{0.5}$ was measured using the raised volume rapid thoracic compression technique [11], after physiotherapy was performed. The other visit was for the deposition study. The breathing circuit was arranged to enable the infants to inhale the aerosol in the supine position. The aerosol emerging from the "infant" circuit had a MMAD of $3.0 \mu \mathrm{m}$ and a GSD of 2.1, i.e. the aerosol had similar characteristics to that from the circuit for the older children.

A transmission scan was performed on one visit, before aerosol inhalation, to determine lung outline and to allow correction for lung attenuation [12]. The subject lay on the tomography bed with a flood source beneath it; prone and supine lung scans were obtained and the geometric mean of the counts calculated. Another scan was obtained without the subject. The square root of the ratio of the counts obtained without the subject to the geometric mean of the counts with the subject provided the attenuation correction factor.

Four $\mathrm{ml}$ of $0.9 \%$ saline with $15 \mathrm{mCi}$ of 99mTc-DTPA were placed in a nebulizer. The nebulizer was driven by compressed air driven at $9 \mathrm{l} \cdot \mathrm{min}^{-1}$. Infants inhaled this aerosol during tidal breathing in the supine position via a mask with a good seal for only $2 \mathrm{~min}$, as longer inhalations tended to cause them to stir. Whether or not the mouth was open during nebulization was recorded.

\section{Protocol for older children}

On one visit, a mouthpiece was used, and on another a mask with a good seal was used with nose-breathing with mouth closed. On each visit, physiotherapy was performed before baseline $\mathrm{FEV}_{1}$ was measured with a Morgan spirometer (Morgan Instruments, Andover, MA, USA) [13]. A transmission scan was obtained as described for the infants, before aerosol inhalation.

Four $\mathrm{ml}$ of the radiolabelled $0.9 \%$ saline were placed in a nebulizer. The nebulizer was driven by compressed air at $9 l \cdot \mathrm{min}^{-1}$. Subjects inhaled this aerosol with tidal breathing in the sitting position for $4 \mathrm{~min}$. No attempt was made to control breathing pattern. For all studies, immediately following the aerosol inhalation, a mouth gargle was performed.

\section{Protocol for infants and older children after nebulization}

Planar scans of the pharynx, lungs (prone and supine scans) and stomach were then obtained by gamma camera (Siemens Gamma Sonics, Inc., Des Plaines, Il, USA) and the deposition images stored on computer. The lung outline from the transmission scan was superimposed on the planar deposition images for analysis.

Three-dimensional distribution in lungs was assessed using single-photon emission computed tomography (SPECT) scanning; 64 views were obtained by rotating the gamma camera $360^{\circ}$ around the subject, which took $16 \mathrm{~min}$.

The breathing circuit was dismantled, sealed and the parts placed in templates, which were then put on the gamma camera for counting. The nebulizer and absolute filter were counted on the following day, as the activity was too high to be counted accurately by the camera on the study day. All counts were corrected for decay. Activity was calculated using a calibration factor of 6,475 counts per second (cps) $\cdot \mathrm{mCi}^{-1}$, which was derived by obtaining the counts on the gamma camera of a range of known activity measured in the dose calibrator. The radioactivity output by the nebulizer was the difference between the original activity of the solution placed in the nebulizer bowl and the residual activity remaining after nebulization. 


\section{Total lung deposition}

The counts were obtained from the planar scans. The trachea was not included, because activity in the oesophagus could not be differentiated from activity in the trachea. Absolute activity in the lungs was derived from the equation by MaceY and Marshall [12]: $\mathrm{T}=\mathrm{G} / \mathrm{E}$ $\sqrt{ }\left(\mathrm{N}_{0} / \mathrm{N}_{\mathrm{t}}\right)$; where $\mathrm{T}=$ activity in lungs $(\mathrm{mCi}), \mathrm{G}=$ geometric mean of counts $\cdot \mathrm{s}^{-1}$ corrected for background and decay (cps), $\mathrm{E}=$ efficiency factor of camera and collimator (cps. $\left.\mathrm{mCi}^{-1}\right), \sqrt{ }\left(\mathrm{N}_{0} / \mathrm{N}_{\mathrm{t}}\right)$ = attenuation correction factor (where No $=$ cps with no subject, and $\mathrm{N}_{\mathrm{t}}=$ cps with subject). Total deposition was expressed as a percentage of nebulizer output and not as absolute activity, because nebulizer output is variable [14].

\section{Regional lung deposition}

Two penetration indices were obtained: one from the planar scans, and the other from the SPECT scans. The penetration index (PI) was defined as the ratio of cps.pixel ${ }^{-1}$ in the peripheral region to that in the central region. The peripheral region was a peripheral rim, the thickness of which was 0.2 of the lung height. The central region at the medial border was 0.5 of the lung height and 0.5 of the lung width [15]. The right lung was used to calculate the PI. The left lung was not used, because the activity in the stomach would affect the PI [15]. The trachea was excluded, because activity in the oesophagus would also affect the PI.
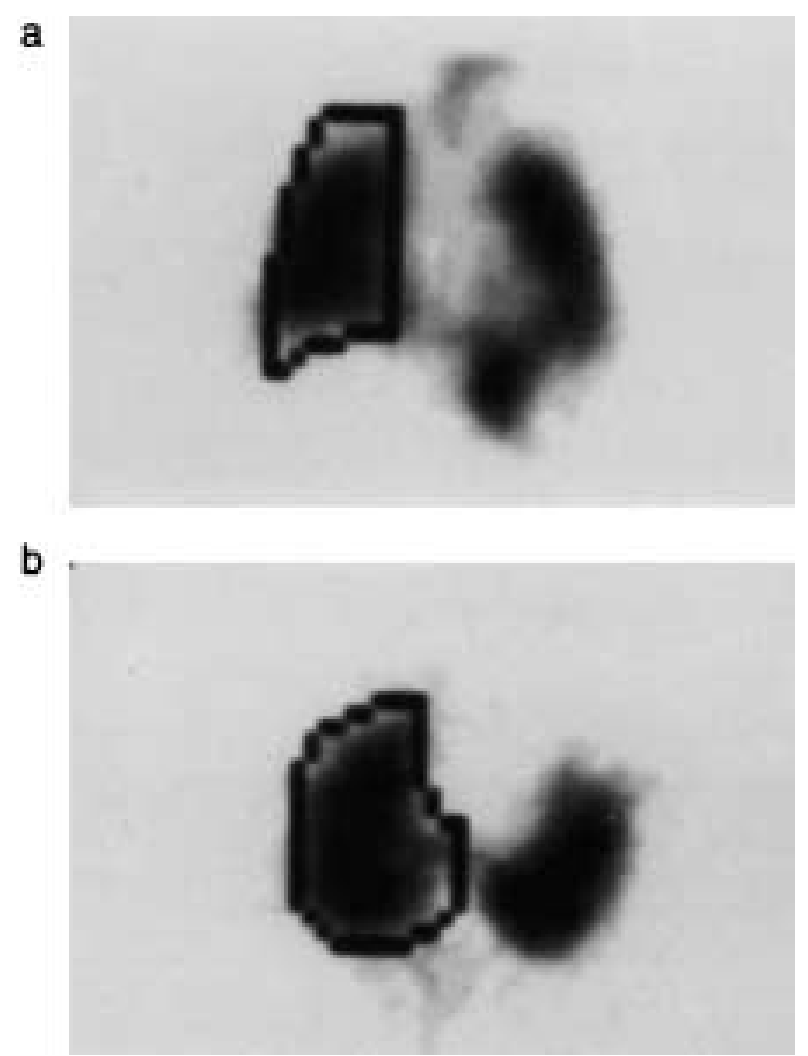

Fig. 1. - Reconstructed a) coronal and b) transverse images of singlephoton emission computed tomography (SPECT) scan on subject No. 14 for aerosol inhaled via the nasal route. Right lung is outlined.
The coronal and transverse images of the SPECT scans were reconstructed (fig. 1). The PI was obtained from the central slice (equal to 0.4 of the lung depth for the coronal slice, and 0.5 of the lung height for the transverse slice) [15].

The absolute activity in the central and peripheral regions of the planar scans was derived proportionately from the counts in these regions and the counts and absolute activity in the right lung.

Deposition in the upper respiratory tract, oesophagus and stomach ("head") was obtained by subtraction: activity placed in nebulizer minus activity in equipment postnebulization minus lung activity.

Polynomial curves were plotted with the best-fit polynomial regression equation. Statistical significance was accepted at the $5 \%$ level.

\section{Results}

The results of the studies are presented in table 1. A total of 28 deposition studies were performed on 20 subjects.

\section{Inhalation via the nasal route}

Twenty studies were carried out on the 12 infants and eight older children. We found that the infants inhaled via the nasal route, whether or not the mouth was open, because the scans did not reveal deposition in the oral cavity. There was an effect of age on total lung deposition (fig. 2): the median lung deposition was $1.3 \%$

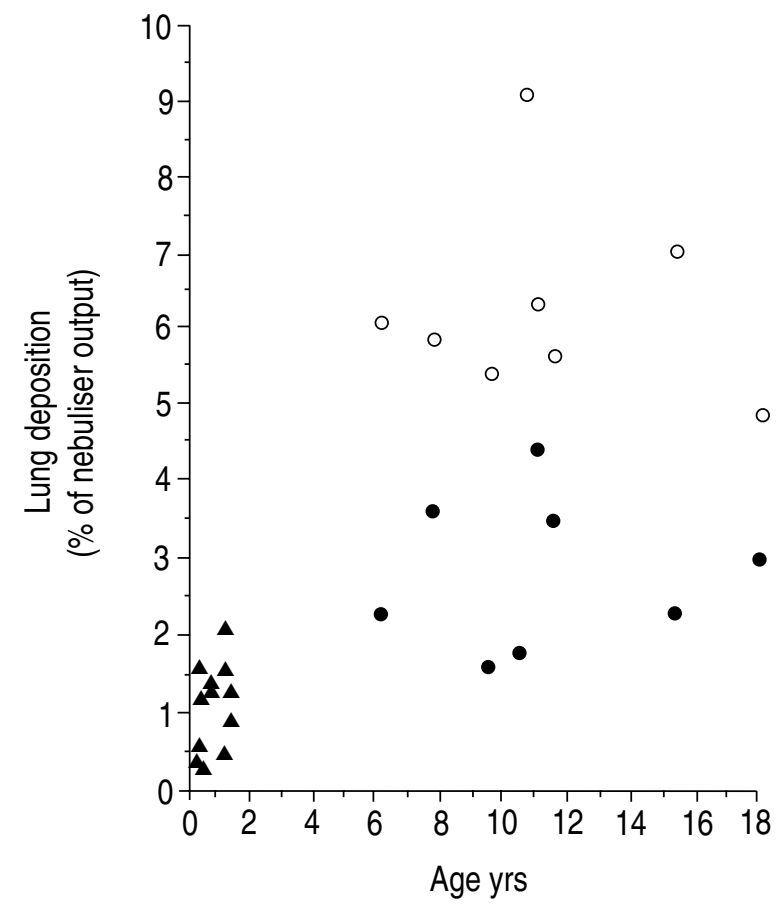

Fig. 2. - The effect of age on lung deposition for aerosol inhaled via the nasal and oral route. $\boldsymbol{\Delta}$ : infants via nasal route; $\boldsymbol{O}$ : older children via nasal route; $\bigcirc$ : older children via oral route. Median lung deposition for nasal inhalation was $1.3 \%$ (range $0.3-1.6 \%$ ) in infants compared to $2.7 \%$ (range $1.6-4.4 \%$ ) in older children. Median lung deposition for oral inhalation was $6.0 \%$ (range $4.9-9.1 \%$ ) in older children. 
Table 1. - Deposition data for nose breathing and mouth breathing

\begin{tabular}{|c|c|c|c|c|c|c|c|c|}
\hline \multirow[t]{2}{*}{$\begin{array}{l}\text { Subject } \\
\text { No. }\end{array}$} & Age & $\begin{array}{l}\text { "Head" \& } \\
\text { lung }\end{array}$ & "Head" & Lung & RAc & RAp & PI pl & PI SP \\
\hline & yrs & $\%$ & $\%$ & $\%$ & $\%$ & $\%$ & & \\
\hline \multicolumn{9}{|c|}{ Nose breathers } \\
\hline 1 & 0.3 & 71.5 & 71.1 & 0.4 & 0.12 & 0.05 & 0.49 & 0.45 \\
\hline 2 & 0.3 & 45.1 & 44.5 & 0.6 & 0.19 & 0.07 & 0.39 & 0.58 \\
\hline 3 & 0.3 & 50.0 & 48.4 & 1.6 & 0.43 & 0.27 & 0.42 & 0.43 \\
\hline 4 & 0.4 & 85.0 & 84.7 & 0.3 & 0.07 & 0.04 & 0.51 & 0.50 \\
\hline 5 & 0.5 & 44.2 & 43.0 & 1.2 & 0.31 & 0.19 & 0.48 & 0.44 \\
\hline 6 & 0.8 & 58.6 & 57.3 & 1.3 & 0.30 & 0.19 & 0.64 & 0.53 \\
\hline 7 & 0.8 & 42.5 & 41.1 & 1.4 & 0.38 & 0.18 & 0.43 & 0.57 \\
\hline 8 & 1.2 & - & - & 0.5 & 0.08 & 0.08 & 0.76 & - \\
\hline 9 & 1.2 & 42.7 & 40.6 & 2.1 & 0.49 & 0.29 & 0.58 & 0.63 \\
\hline 10 & 1.2 & 48.4 & 46.8 & 1.6 & 0.53 & 0.32 & 0.54 & 0.55 \\
\hline 11 & 1.4 & 70.3 & 69.0 & 1.3 & 0.33 & 0.17 & 0.47 & 0.48 \\
\hline 12 & 1.4 & 40.7 & 38.8 & 0.9 & 0.51 & 0.51 & 0.48 & 0.55 \\
\hline 13 & 6.3 & - & - & 2.3 & 0.48 & 0.52 & 0.67 & 0.46 \\
\hline 14 & 7.5 & 63.0 & 59.4 & 3.6 & 1.04 & 0.68 & 0.72 & 0.67 \\
\hline 15 & 9.5 & 70.9 & 69.3 & 1.6 & 0.36 & 0.27 & 0.70 & - \\
\hline 16 & 10.5 & 46.0 & 44.2 & 1.8 & 0.43 & 0.35 & 0.64 & 0.65 \\
\hline 17 & 11.0 & 24.1 & 19.7 & 4.4 & 1.20 & 0.60 & 0.49 & 0.53 \\
\hline 18 & 11.5 & 60.4 & 56.9 & 3.5 & 0.94 & 0.56 & 0.55 & - \\
\hline 19 & 15.3 & 32.8 & 30.5 & 2.3 & 0.75 & 0.22 & 0.29 & 0.53 \\
\hline 20 & 18.0 & 66.5 & 63.5 & 3.0 & 0.62 & 0.37 & 0.56 & 0.55 \\
\hline \multicolumn{9}{|c|}{ Mouth breathers } \\
\hline 13 & 6.3 & 43.1 & 37.0 & 6.1 & 1.40 & 1.37 & 0.60 & 0.53 \\
\hline 14 & 7.5 & 42.1 & 36.2 & 5.9 & 1.89 & 1.09 & 0.54 & 0.52 \\
\hline 15 & 9.5 & 54.7 & 49.4 & 5.3 & 1.29 & 0.79 & 0.59 & 0.54 \\
\hline 16 & 10.5 & - & - & 9.1 & 2.28 & 1.50 & 0.55 & 0.39 \\
\hline 17 & 11.0 & - & - & 6.4 & 1.82 & 1.07 & 0.58 & - \\
\hline 18 & 11.5 & 35.7 & 30.1 & 5.6 & 1.55 & 0.99 & 0.57 & 0.53 \\
\hline 19 & 15.3 & 40.5 & 33.4 & 7.1 & 2.00 & 0.95 & 0.49 & 0.53 \\
\hline 20 & 18.0 & 20.5 & 15.6 & 4.9 & 1.00 & 0.59 & 0.57 & 0.65 \\
\hline
\end{tabular}

"Head": calculated upper respiratory tract, oesophagus and stomach activity expressed as a percentage of nebulizer output; Lung: activity in the lungs expressed as a percentage of nebulizer output; RAc: activity in central region expressed as a percentage of nebulizer output; RAp: activity in peripheral region expressed as a percentage of nebulizer output; PI pl: penetration index from planar scan; PI SP: penetration index from single-photon emission computed tomography (SPECT) scan; -: data missing due to technical difficulties.

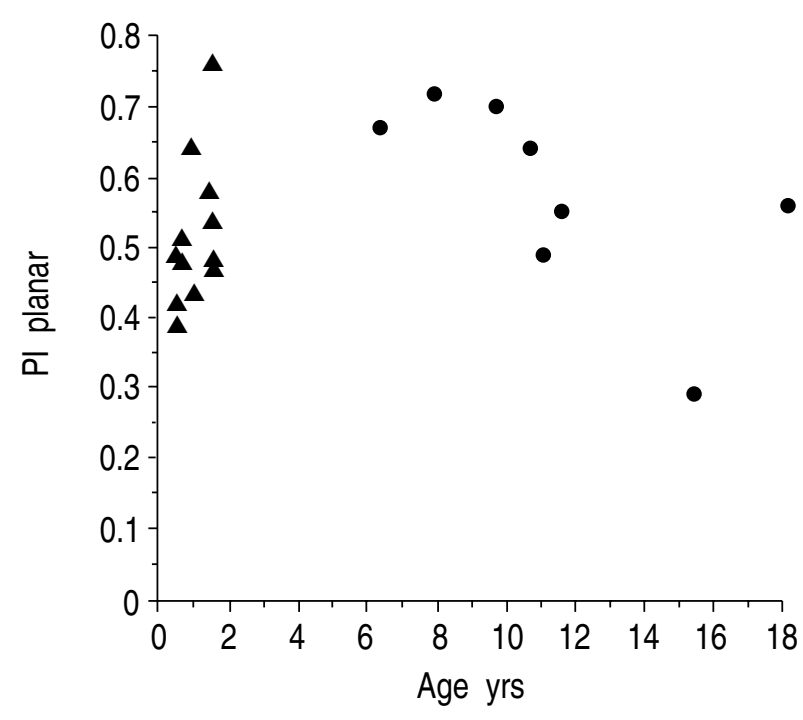

Fig. 3. - Penetration index (PI) from planar scans for aerosol inhaled via the nasal route. $\mathbf{\Delta}$ : infants; $\boldsymbol{O}$ : older children.

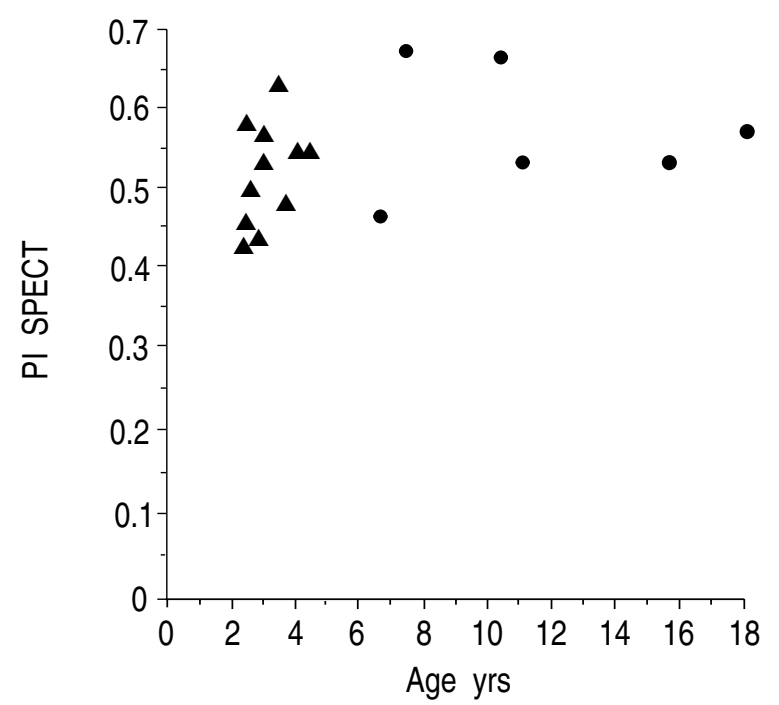

Fig. 4. - Penetration index (PI) from single-photon emission computed tomography (SPECT) scans for aerosol inhaled via the nasal route. infants; O: older children. 
(range $0.3-1.6 \%$ ) in infants compared to $2.7 \%$ (range $1.6-4.4 \%)$ in older children $(\mathrm{p}=0.0001)$. The relationship between total lung deposition and age was similar to that between lung deposition and weight $(\mathrm{p}=0.0002)$, height $(\mathrm{p}=0.0001)$ and body surface area $(\mathrm{p}=0.0001)$. There was a similar relationship between age and deposition both for central $(\mathrm{p}=0.0008)$ and peripheral $(\mathrm{p}=0.005)$ deposition. There was no significant effect of age on upper respiratory tract, or the sum of upper respiratory tract and lung deposition.

Aerosol deposition from calculating penetration indices from planar (PI pl) and SPECT (PI SP) scans did not change significantly with age (figs. 3 and 4 ). The median PI pl for the infants was 0.49 (range 0.39-0.76) compared to a median of 0.63 (range $0.29-0.70$ ) for the older children. The corresponding values for PI SP were 0.53 (range 0.43-0.63) and 0.54 (range 0.46-0.67), respectively. There was no correlation between percentage predicted $\mathrm{FEV}_{0.5}$ and $\mathrm{FEV}_{1}$ and lung deposition.

When the infants and older children were analysed separately, there was no influence of age on lung, upper respiratory tract, or total deposition.

\section{Inhalation via the oral route}

Studies were performed on eight older children (table 1). There was no influence of age on lung (fig. 2), upper respiratory tract, or the sum of upper respiratory tract and lung deposition.

\section{Discussion}

We have established that the dose of a nasally inspired aerosol reaching the lungs in infants who are asleep is approximately half that for older children, when the nebulizer is operating at $9 l \cdot \mathrm{min}^{-1}$. Age does not affect deposition of nasally inspired aerosols in infants as a group, nor does it affect deposition of nasally or orally inspired aerosols in older children.

Previous data in this area have been limited to in vitro studies derived from calculations from morphometric measurements, an in vivo study with potential methodological problems, and an in vivo study in older children. An example of an in vitro study is the work of $\mathrm{YU}$ and $\mathrm{XU}_{\mathrm{U}}$ [3], who used an anatomical lung model formulated mathematically from available physiological and morphmetric data. They predicted that lung deposition would increase from birth, peak at 2 yrs of age and then gradually decline. Since many assumptions are involved in analyses such as these, their validity is difficult to assess. In their in vivo study, ALDERSON et al. [16] assessed radioisotopic aerosol deposition in 11 children with cystic fibrosis, and found a linear relationship between lung deposition and age. The age range studied was not broad: the youngest child was 1.1 yrs compared to $0.3 \mathrm{yr}$ in the present study, and it has been calculated that the dose inhaled at the airway opening increases with age only in the first year of life [2]. The route of inhalation was not noted in the study by ALDERSON et al. [16]; some of their subjects may have inhaled nasally and some may have inhaled orally, and it is difficult to assess the effect of this on the results because the route of inhalation will affect lung deposition [17]. The apparent discrepancy in the findings in the older children between the study by ALDERSON et $a l$. [16] and the present study may be due to the relatively small number of subjects in both studies. O'DOHERTY et al. [18] reported lung deposition of radiolabelled nebulized pentamidine in six children aged 8-11 yrs, six teenagers aged $12-15$ yrs and six adults aged $\geq 18$ yrs with human immunodeficiency virus infection. The subjects inhaled the aerosol from a mouthpiece for 40 min. Mean lung deposition as a percentage of nebulizer output was similar in the children, teenagers and adults. The findings of O'DOHERTY et al. [18] concur with the findings on older children in the present study.

In the current study, we aimed to assess deposition using a commercially available nebulizer run as it would be in a typical clinical or research application. We chose the Turret nebulizer because of its excellent output and droplet characteristics $[10,19]$. It was driven at $9 l \cdot \mathrm{min}^{-1}$ to obtain a respirable aerosol [10]. Other nebulizers and circuits are likely to provide different values for deposition. Since the Turret device generates relatively small droplets, nasal and central airway deposition should be less and peripheral deposition more with this device than with most other nebulizers. The Turret nebulizer was driven at $9 l \cdot \mathrm{min}^{-1}$ for all subjects, because the aim was to assess deposition in routine clinical practice. If driving pressures are adjusted according to age, a low driving pressure would be required for infants, and this would result in aerosol particles which are less respirable.

For nasally inspired aerosols, lung deposition was lower in infants than in older children. This could be due to infants having lower inspiratory flows and, thus, inhaling only part of the nebulizer output, in contrast to the older children who would be inhaling the entire nebulizer output [2]. There was no effect of age on lung deposition when the infants and older children were analysed separately. The findings for the infants are different from those of Collis et al. [2], who calculated that the dose inhaled at the airway opening increases with age in the sleeping infant in the first year of life. The findings for the older children are consistent with the predictions of Collis et al. [2] for older children. The apparent discrepancy in the findings for the infants between the present study and the study by Collis et al. [2] may be due to methodological differences: Collis et al. [2] measured inspiratory flows and calculated the dose inhaled at the airway opening. We hypothesize that the reduced lung deposition in infants is due to the smaller noses of infants acting as better filters.

The data on orally inspired aerosol from the present study demonstrated that there was no significant change in lung, upper respiratory tract, and the sum of upper respiratory tract and lung deposition between 6 and 18 yrs. These data are also in accordance with the predictions of Collis et al. [2]. However, the number of subjects aged 6-18 yrs was small.

In the current study, aerosol distribution was not affected by age. From available knowledge of factors 
affecting distribution of deposition, predictions of the effect of age on deposition would be difficult. For example, smaller airways in younger children would favour central deposition by impaction, but the lower flows would decrease impaction in central airways, and decreased residence time would tend to decrease peripheral deposition by reducing diffusion and sedimentation. The infants inhaled the aerosol in the supine position, and this position has been shown to result in more uniform distribution in the lungs compared to the upright position [20, 21]. Therefore, if infants have relatively central distribution when they inhale aerosol in the erect posture, this may not be demonstrated when the aerosol is administered in the supine position.

A finite amount of clearance of radiolabel will occur during the time taken to perform the SPECT scan. The mean elimination constant for $99 \mathrm{mTc}-\mathrm{DTPA}$ in normal subjects is $0.8 \% \cdot \mathrm{min}^{-1}$, and clearance is faster from the peripheral region compared to the central region of the lung [22]. However, the planar scans which were obtained immediately after inhalation of radioaerosol did not show more peripheral deposition compared to the SPECT scans which were obtained following the planar scans; this suggests that peripheral clearance during the SPECT scans was not significant.

No correlation between nasal deposition and age was found in this study. The study of nasal deposition by BECQUEMIN et al. [23] on children aged more than $5 \mathrm{yrs}$ arrived at the same conclusion. An explanation for these findings is that the lower inspiratory flows in infants limit the mass of aerosol entering the nose, but this is counteracted by the smaller noses of infants being more efficient filters. $\mathrm{YU}_{\mathrm{U}}$ and $\mathrm{XU}_{\mathrm{U}}$ [3] predicted that nasal deposition is greatest at birth and decreases with age. The findings of the present study may also be compatible with theirs, as their calculations were based on a given mass of aerosol entering the nostrils, and, as noted, the infants in the current study are likely to have inspired a lower aerosol mass than older subjects [2]. However, the deposition values for the upper respiratory tract were very high in a number of subjects.

Deposition in the upper respiratory tract was not measured directly, but was obtained by subtraction. This means that the nasal deposition data would have been affected by the cumulative errors inherent in the radionuclide technique. Attenuation correction of the counts obtained in the upper respiratory tract would allow more accurate determination, but a transmission scan of the upper respiratory tract would have incurred additional radiation exposure to the subject. The cumulative errors include: 1) variation in the measurement of the radioactive dose; 2) variation in the rate of clearance of radiolabel [22]; 3) variation in the measurement of counts by the gamma camera [14]; 4) variation in the methods used to calculate deposition (including method used for correcting tissue attenuation) [14]; and 5) intersubject variability [24, 25].

As the subjects in the current study had cystic fibrosis, the data on the influence of age obtained for these children may not simulate data which would be obtained in normal subjects. However, most of the subjects had normal or supranormal lung function, and none had nasal polyps or were unwell when the studies were performed.

This data should be of use in helping to formulate more rational dosage schedules for inhaled drugs in children. As lung deposition does not increase between 6 and 18 yrs, a 6 year old child would, therefore, have the same lung deposition as an 18 year old for a given nebuliser dose; this could mean that a 6 year old child would be more likely to experience side-effects for toxic agents. Therefore, in order to deposit a given mass of drug in a given volume of lung, nebulizer solution drug concentrations need to be directly related to the size of the child. Similarly, nebulizer solution drug concentrations should be directly related to the size of an infant aged up to $1.5 \mathrm{yrs}$. However, the infant who is asleep will receive only half as much drug for a given volume of lung, and this could account for some treatment failures. Therefore, nebulizer solution drug concentrations which are directly related to the size of the infant need to be doubled. Further studies need to be performed to determine if this conclusion is applicable to infants who are awake. Children between 1.5-6 yrs need to be studied before recommendations on dosage adjustments can be made. However, these studies are likely to be difficult to perform in infants who are awake and children aged between 1.5 and 6 years because of the lack of cooperation. Further studies with children with cystic fibrosis who have severe lung damage may be necessary, as aerosol deposition and distribution are likely to be affected by the degree of lung involvement.

Acknowledgments: The authors thank D. Turner for infant pulmonary function testing, D. Burton and S. Walker for spirometry, and D. Konstant for particle sizing. HLC was supported by Telethon, Australian Cystic Fibrosis Association, and $\mathrm{NH} \& \mathrm{MRC}$ grants.

\section{References}

1. Sly PD, Le Souef PN. Inhaled therapy in paediatrics. $J$ Paediatr Child Health 1991; 27: 7-10.

2. Collis GG, Cole CH, Le Souef PN. Dilution of nebulised aerosols by air entrainment in children. Lancet 1990; 336: 341-343.

3. $\mathrm{Yu} \mathrm{CP}, \mathrm{Xu}$ GB. Predicted deposition of diesel particles in young humans. J Aerosol Sci 1987; 18(4): 419-429.

4. Phalen RF, Oldham MJ, Beaucage CB, Crocker TT, Mortensen JD. Postnatal enlargement of human tracheobronchial airways and implications for particle deposition. Anat Rec 1985; 212: 368-380.

5. Phalen RF, Oldham MJ, Kleinman MT, Crocker TT. Tracheobronchial deposition predictions for infants, children and adolescents. Ann Occup Hyg 1988; 32 (suppl. 1): 11-21.

6. Hofmann W. Dose calculations for the respiratory tract from inhaled natural radioactive nuclides as a function of age. - II: Basal cell dose distributions and associated lung cancer risk. Health Physics 1982; 43(1): 31-44.

7. Hofmann W, Martonen TB, Graham RC. Predicted deposition of nonhygroscopic aerosols in the human 
lung as a function of subject age. J Aerosol Med 1989; 2(1): 49-68.

8. Hubbard RC, McElvaney NG, Birrer P, et al. A preliminary study of aerosolized recombinant human deoxyribonuclease I in the treatment of cystic fibrosis. N Engl J Med 1992; 326(12): 812-815.

9. Crystal RG. Gene therapy strategies for pulmonary disease. Am J Med 1992; 92(6A): 44-52.

10. Newman SP, Woodman G, Clarke SW. Deposition of carbenicillin aerosols in cystic fibrosis: effects of nebuliser system and breathing pattern. Thorax 1988; 43: 318-322.

11. Turner DJ, Stick SM, Le Souef KL, Le Souef PN. Assessment of respiratory function in infants pumped to higher lung volumes (Abstract). Am Rev Respir Dis 1991; 143 (suppl.): A126.

12. Macey DJ, Marshall R. Absolute quantitation of radiotracer uptake in the lungs using a gamma camera. $J \mathrm{Nucl}$ Med 1982; 23: 731-735.

13. Knudson RJ, Lebowitz MD, Holberg CJ, Burrows B. Changes in the normal maximal expiratory flow-volume curve with growth and aging. Am Rev Respir Dis 1983; 127: 725-734.

14. Thomas SHL, O'Doherty MJ, Page CJ, Nunan TO. Variability in the measurement of nebulized aerosol deposition in man. Clin Sci 1991; 81: 767-775.

15. Phipps PR, Gonda I, Bailey DL, Borham P, Bautovich $\mathrm{G}$, Anderson SD. Comparisons of planar and tomographic gamma scintigraphy to measure the penetration index of inhaled aerosols. Am Rev Respir Dis 1989; 139: 1516-1523.

16. Alderson PO, Secker-Walker RH, Strominger DB, Markham J, Hill RL. Pulmonary deposition of aerosols in children with cystic fibrosis. J Pediatr 1974; 84(4): 479-484.

17. Wolfsdorf J, Swift DL, Avery ME. Mist therapy reconsidered; an evaluation of the respiratory deposition of labelled water aerosols produced by jet and ultrasonic nebulizers. Pediatr 1969; 43(5): 799-808.

18. O'Doherty MJ, Thomas SHL, Gibb D et al. Lung deposition of nebulised pentamidine in children. Thorax 1993; 48: 220-226.

19. Newman SP, Pellow PGD, Clarke SW. Choice of nebulisers and compressors for delivery of carbenicillin aerosol. Eur J Respir Dis 1986; 69: 160-168.

20. Baskin Ml, Abd AG, llowite JS. Regional deposition of aerosolized pentamidine: Effects of body position and breathing pattern. Ann Intern Med 1990; 113: $677-$ 683.

21. O'Doherty MJ, Thomas SH, Page CJ, Bradbeer C, Nunan TO, Bateman NT. Does inhalation of pentamidine in the supine position increase deposition in the upper part of the lung? Chest 1990; 97: 1343-1348.

22. Groth S. Pulmonary clearance of ${ }^{99 \mathrm{~m} T \mathrm{Tc}-D T P A}$. Dan Med Bull 1991; 38: 189-203.

23. Becquemin MH, Swift DL, Bouchikhi A, Roy M, Teillac A. Particle deposition and resistance in the noses of adults and children. Eur Respir J 1991; 4: 694-702.

24. Heyder J, Gebhart J, Roth C, et al. Intercomparison of lung deposition data for aerosol particles. J Aerosol Sci 1978; 9: 147-155.

25. Heyder J, Gebhart J, Grein E, Stahlhofen W, Stuck B. Biological variability of particle deposition in the human respiratory tract during controlled and spontaneous mouth-breathing. Ann Occup Hyg 1982; 26: 137-147. 